Research Journal of Applied Sciences, Engineering and Technology 16(5): 175-184, 2019

DOI: $10.19026 /$ rjaset.16.5385

ISSN: 2040-7459; e-ISSN: 2040-7467

(C) 2019 Maxwell Scientific Publication Corp.

Submitted: March 28, 2018

Accepted: April 23, 2018

Published: December 15, 2019

\title{
Research Article \\ Comparison of Some PWM Techniques for Control of a Single-Phase Inverter
}

\author{
${ }^{1}$ Mouhamadou Thiam, ${ }^{1}$ Boussef Sy, ${ }^{1}$ Moustapha Diop, ${ }^{1}$ Alphousseyni Ndiaye, ${ }^{1}$ Samba Gueye, \\ ${ }^{1}$ Ibrahima Ngom and ${ }^{2}$ Ousseynou DIAO \\ ${ }^{1}$ Laboratoire d'Energies Renouvelables (LER) \\ ${ }^{2}$ Laboratoire d'Energétiques Appliquées (LEA)/Ecole Supérieure Polytechnique (ESP) Dakar/ Université \\ Cheikh Anta Diop (UCAD) Dakar Sénégal
}

\begin{abstract}
Single-phase voltage inverters are used among others in relief supplies and DC-DC converters to the alternative middle tier. In these applications, the main purpose is to obtain a sinusoidal AC output signal whose amplitude and frequency can be controlled, as well as the low levels of THD. This document presents a comparative study of certain orders single-phase inverters techniques. The techniques used are studied; the results of the simulations will be discussed.
\end{abstract}

Keywords: Converter, filter, inverter, PWM, THD

\section{INTRODUCTION}

Development policy of local expertise for the mastery of technologies related to the photovoltaic conversion requires the implementation of static models converters such as inverters.

The relevance of this problem is confirmed during the preparatory work for this study: indeed, due to the national electricity grid failures, harmonic currents can give birth to harmonics of voltage distorted mains voltage wave and generate additional losses, mention (Ryan et al. (1997) and Houldsworth and Grant (1984). The harmonics that is still present in a makeup unfit sine wave to use electrical noise is a matter of concern, as in medical devices that monitor the vital signs of a particular human being also in the field of renewable Energies.

These are secondary harmonics that are additional to a main oscillation and whose frequency is a multiple of the main swing. Indeed, one of the major objectives of researchers in power electronics and control is to eliminate this secondary phenomenon which disrupts the electrical network.

The objective is to study the basic control technics most used and some called advanced technologies, evaluate them and select one that would be more appropriate for minimizing harmonics.

This study also tends to demonstrate the performance of certain control technics according to the comparison of parameters obtained from the simulation results.
From the point of view of control inverter, it can be controlled using several techniques; the most widespread is the (PWM) pulse width modulation technique.

This technique allows the static inverter to generate output waves very close to the ideal shape, but also linear amplitude control of output voltage and current with control switches- semiconductors, however the magnitude of the fundamental component output waveform is attenuated and stress on semiconductorswitching components is higher, which reduces the operating time "life of the component", resulting in the highest frequency of switching. What justifies the interest of the comparative study of some of these commands techniques to reduce these constraints, after which their performance assessment will be based on parameters such as the rate of Harmonic Distortion (THD) and the response time of the system.

\section{INVERTER CONSTITUTION}

The inverters consist of active and passive components, sophisticated and powerful that admit however a certain number of limitations that are not without effect on the synthesis of control loops. Shete et al. (2012) in his publication uses of power IGBT will be put forward in order to allow a higher switching frequency. On the other hand, a compromise must be made since a higher switching frequency involves greater losses by switching and the heating of

Corresponding Author: Mouhamadou Thiam, Laboratoire d'Energies Renouvelables (LER)/Ecole Supérieure Polytechnique (ESP) Dakar/Université Cheikh Anta Diop (UCAD) Dakar Sénégal

This work is licensed under a Creative Commons Attribution 4.0 International License (URL: http://creativecommons.org/licenses/by/4.0/). 


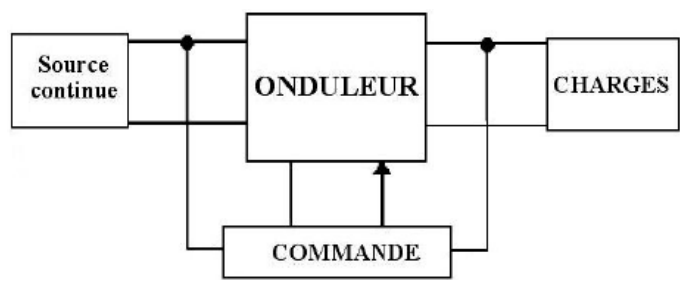

Fig. 1: Diagram of an inverter control

the various components constituting this up and thus a decrease in the efficiency of conversion, proposes (Thangaprakash and Krishnan, 2010). In addition, the final system should have the best possible performance while being the most affordable possible so eventually to make it marketable. It is important to choose those elements; they are closely related. A high yield may result in significant costs and not inconsiderable dimensions (Fig. 1).

The inverters are static power electronics converters. They are used in any electrical system where the transformation of the voltage continues (Vdc) alternating voltage (Vac) is required, (Houldsworth and Grant, 1984).

The basic concept of the inverters is easily understandable and it is independent of the technology used. The central part of a UPS is frequently consisting of a bridge to connect each of the two poles of entry to each of the two poles of output through an electronic switch solid-state. follows:

The three main elements that constitute a ups are as

- Continuous voltage Source (for example: battery)

- $\mathrm{DC} / \mathrm{AC}$ converter

- LC filter

Any approach to command of a UPS should take into account the two following constraints, (Boumaaraf et al., 2002):

- The reduction of overall harmonic distortion in transient and steady-state;

- Access to the setting of the fundamental output voltage to a reference value.

Diagram in Fig. 2 shows the functional structure of a type full-bridge inverter.

This bridge, which switches according to the cadence of the frequency $(50 \mathrm{~Hz})$, allows alternative loads. However, it would be a current rectangular whose intensity is not influenced at the output of the switch. In order to have a pure sine wave, two types of filters are used: inductive (for current) and capacitive (for tension).

Control strategies: In practice, many industrial applications receive their power from a power source

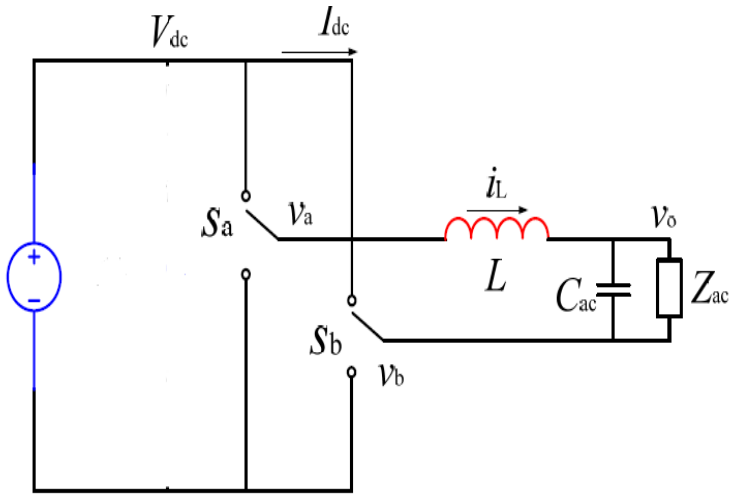

Fig. 2: Summary of a full bridge inverter

that is stable and adjustable. This tension is often obtained with inverters which eliminate the continuous input voltage variations and maintain constant the relationship voltage/frequency while adjusting the amplitude of the voltage required by the load, (Chikhi and Chikhi, 2002). Several methods are used to obtain the desired voltage such that the control full wave commonly referred to as symmetrical control, the offset command and another very effective is called "Pulse Width Modulation: (PWM). In addition to adjust the amplitude, this method controls the harmonic content of the output voltage inverter, pushing the low-order harmonics towards higher frequencies. This allows easier and inexpensive voltage filtering, because the size of the filter components is smaller. It is important to mention that the PWM technique has limitations compared with the frequency of operation of the inverters; higher is the frequency. More important are the losses due to the switching of semiconductor switches; the same constraints in these devices are also high, their life expectancy is reduced. The PWM inverters operating frequency is also limited by the own switches switching speed Semiconductor. In this study, we will present and examine in detail the PWM command.

Principle and characteristics of the pulse width modulation: Conversion continues-alternative is provided by switching the inverter switches that will make temporary connections between the terminals of the continuous power supply and alternative care. The transfer of energy is controlled by the ratio between opening and closing (duty cycle) of each switch intervals, so by the control of these switches (MLI) pulse width modulation, this technique is based on the comparison of a reference signal called also modulator amplitude Ar and frequency fr, with a known signal carrier Ap amplitude and frequency fp high.

A single-phase Inverter control techniques: There are several techniques to vary the gain of an inverter; the most effective are those at PWM mention (Shete et al., 
2012). The techniques the most widespread in this area are: simple linear modulation, multiple linear modulation, modulation triangular sine, modified sine triangular Modulation

Simple linear modulation: This PWM technique uses a single pulse by half and the width of the pulse varies the amplitude of the voltage at the output of the inverter (to the load terminals). The control signals are obtained by comparison to a reference signal rectangular of amplitude Ar with a triangular carrier wave of amplitude signal Ap. The frequency of the reference signal is the fundamental of the output voltage. Figure 3 shows these two signals and the shape of the output voltage for Inverter Bridge.

By varying from 0 to $\mathrm{Ap} \mathrm{Ar}$, pulse width can vary from 0 to $180^{\circ}$. The relationship between $\mathrm{Ar}$ and Ap is the control variable and is called amplitude modulation index or just simply modulation index.

Multiple linear modulation: When we want to reduce the harmonic content, it uses several pulses in each alternations of the output voltage. This technique is known as the multiple PWM. The switches control pulses are obtained in a similar way to that of the

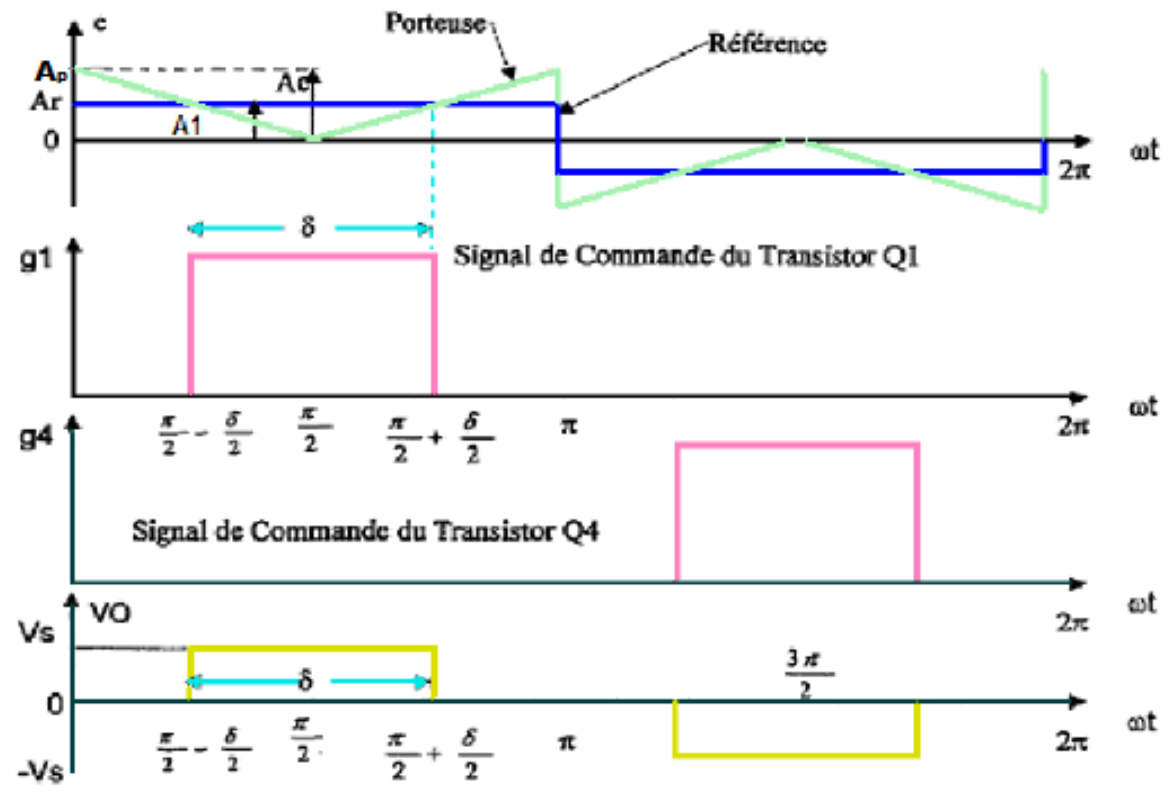

Fig. 3: Simple sinusoidal pulse width modulation
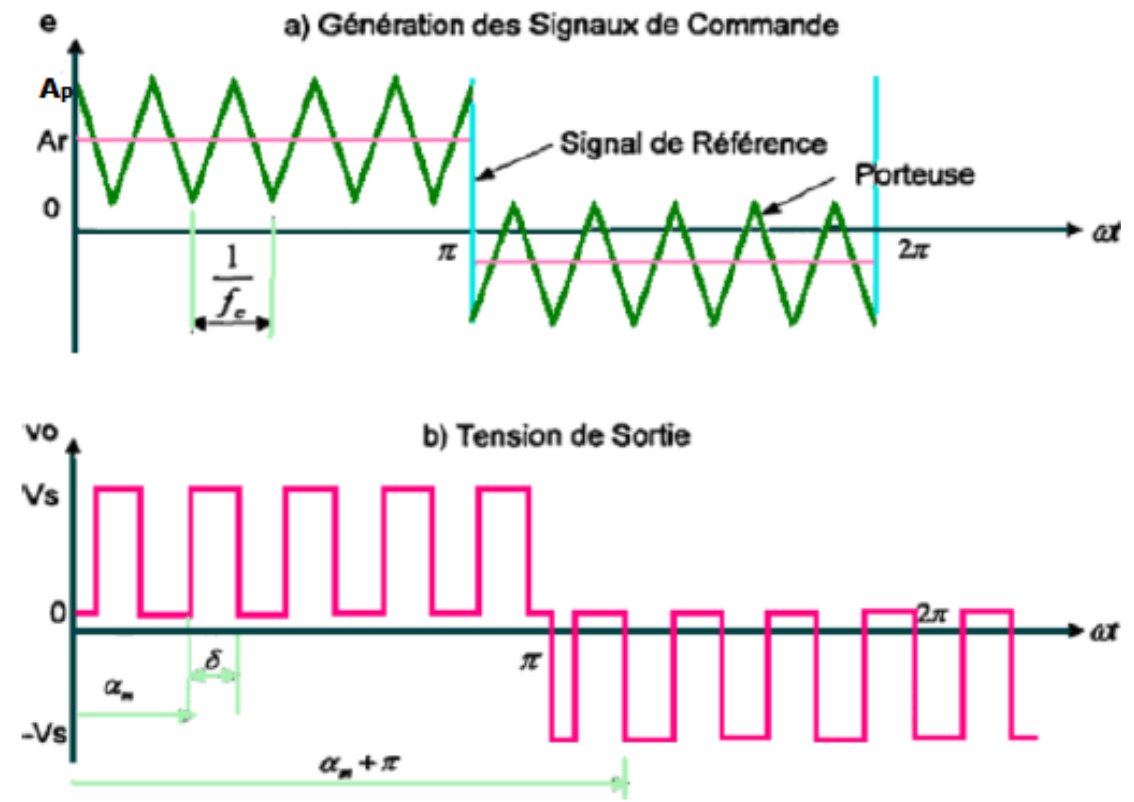

Fig. 4: Multiple sinusoidal pulse width modulation 
previous technique, with the intersections of a signal of reference and a carrier signal (Fig. 4). The frequency of the carrier signal is higher than the frequency of the reference signal, what is the main difference of this technique is that it generates more than a pulse to the output. The reference rule in output frequency signal frequency and the carrier frequency fp of the signal determines the number of pulses during the half alternation.

The modulation index controls the amplitude of the output voltage.

This type of modulation is also known as the modulation pulse uniform (UPWM 'Uniform Pulse Width Modulation') width. The number of pulses per half cycle is:

$$
\mathrm{p}=\frac{\mathrm{fp}}{2 \mathrm{fr}}=\frac{m_{f}}{2}
$$

Hence $m_{f}=\frac{\mathrm{fp}}{\mathrm{fr}}$ is called rate of frequency modulation.

Sine triangular modulation: This technique is similar to the previous technique, but the reference signal is a sinusoidal signal. This change gives as a result of the pulses of different widths, proportional to the amplitude of the wave of reference (Shete et al., 2012). The switches control signals are always generated by the intersection of a triangular carrying signal of frequency fp with a sinusoidal signal frequency fr. reference Fig. 5 shows the output voltage.

Reference signal frequency fr, determines the frequency of the output signal from the inverter, while the maximum amplitude control modulation index, $m$ $f$., which in turn controls the effective output voltage.

The effective output voltage can be varied by varying the modulation index, $m-f \ldots$ One can observe that the area of each pulse corresponds approximately to the area above the sine wave between the half of the adjacent points of the period at the beginning control signals. Sine triangular modulation amended.

According to the characteristic of the sinusoidal PWM, the widths of the pulses approach the maximum amplitude of the sine wave to not significantly change with the change of the modulation index (Fig. 6). This is due to the characteristics of a sine wave and sine wave PWM technique can be changed by applying the sine wave during the beginning and end of an interval of $60^{\circ}$ by [amp] frac 12 ; it is to say 0 to $60^{\circ}$ and $120^{\circ}$ to $180^{\circ}$.

The number of pulses $\mathrm{p}$ on half a $60^{\circ}$ period is normally linked to the frequency ratio in the case of a three-phase inverter by the equation:
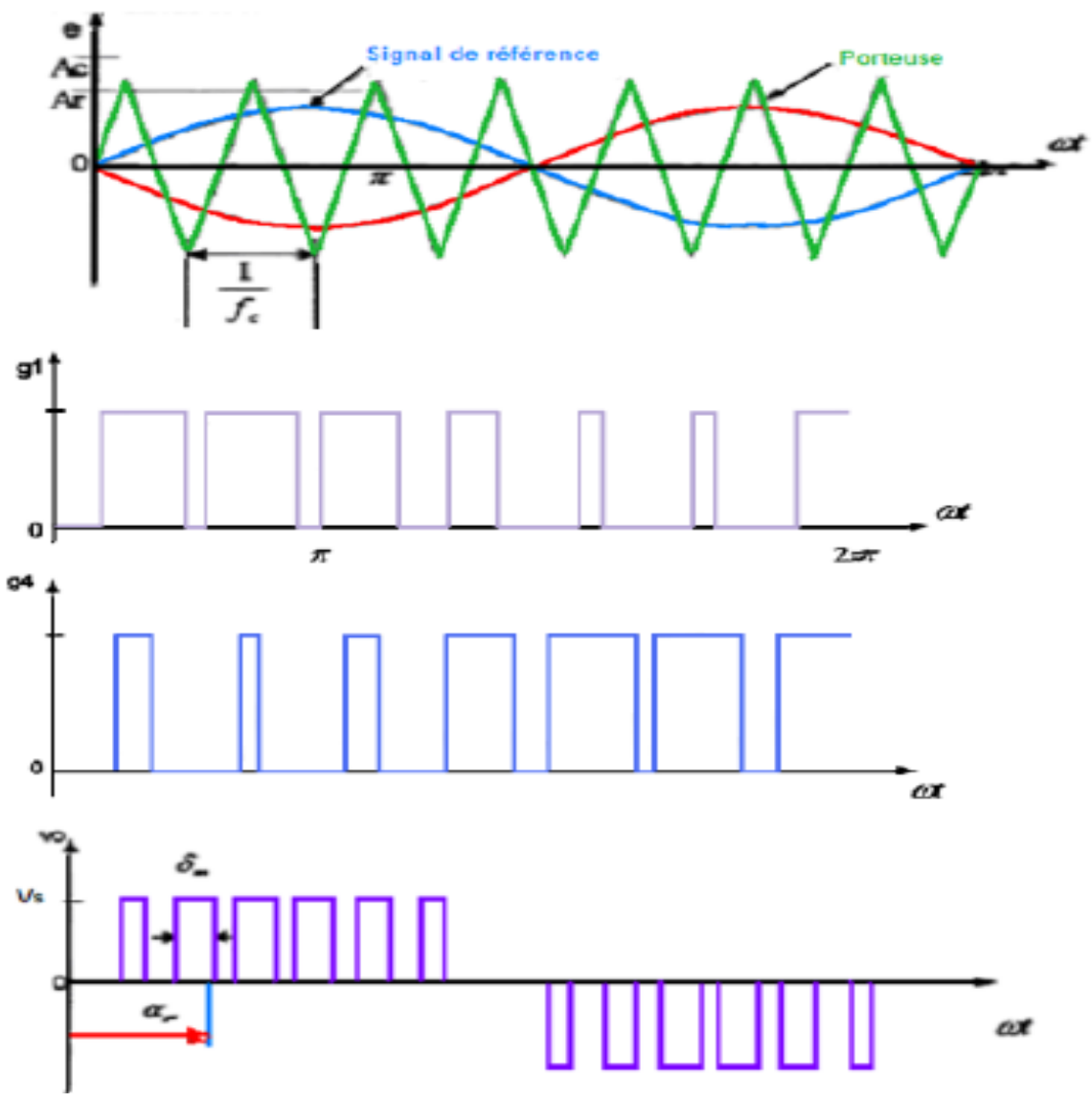

Fig. 5: Sinusoidal pulse width modulation 


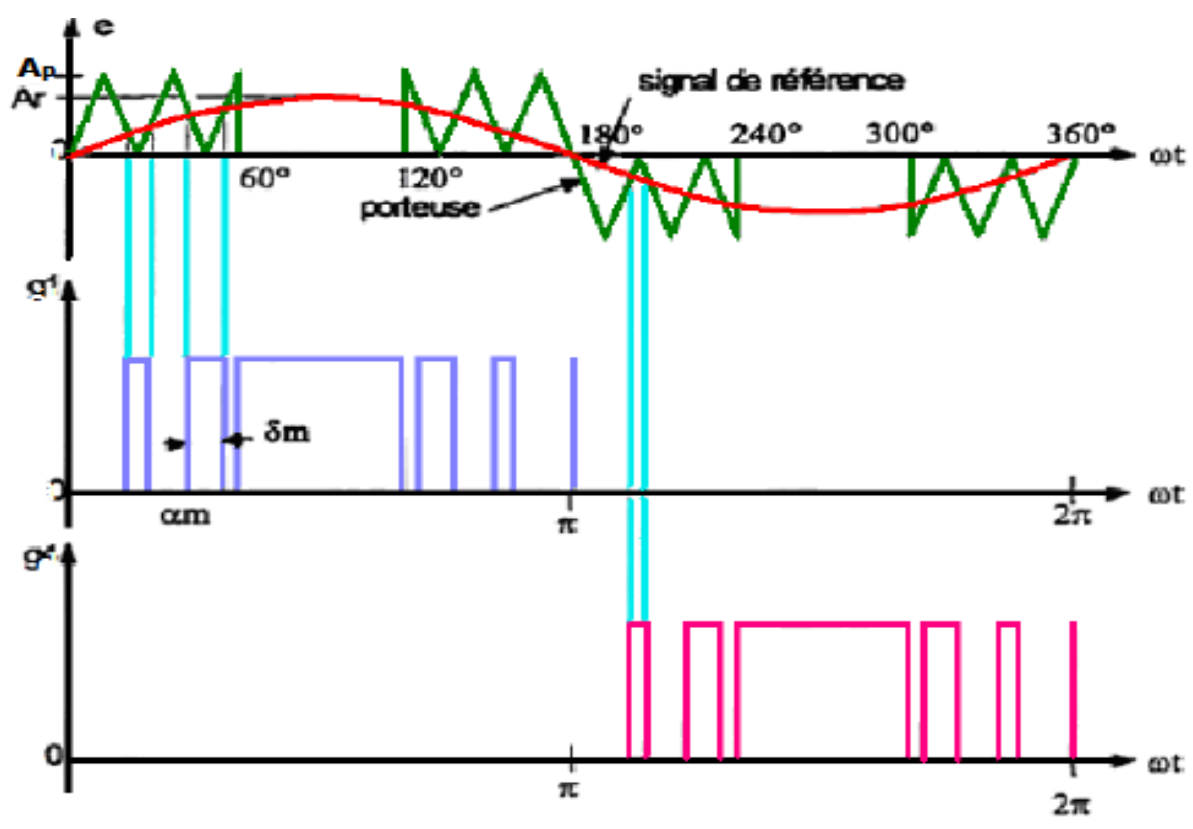

Fig. 6: Modified sine pulse width modulation

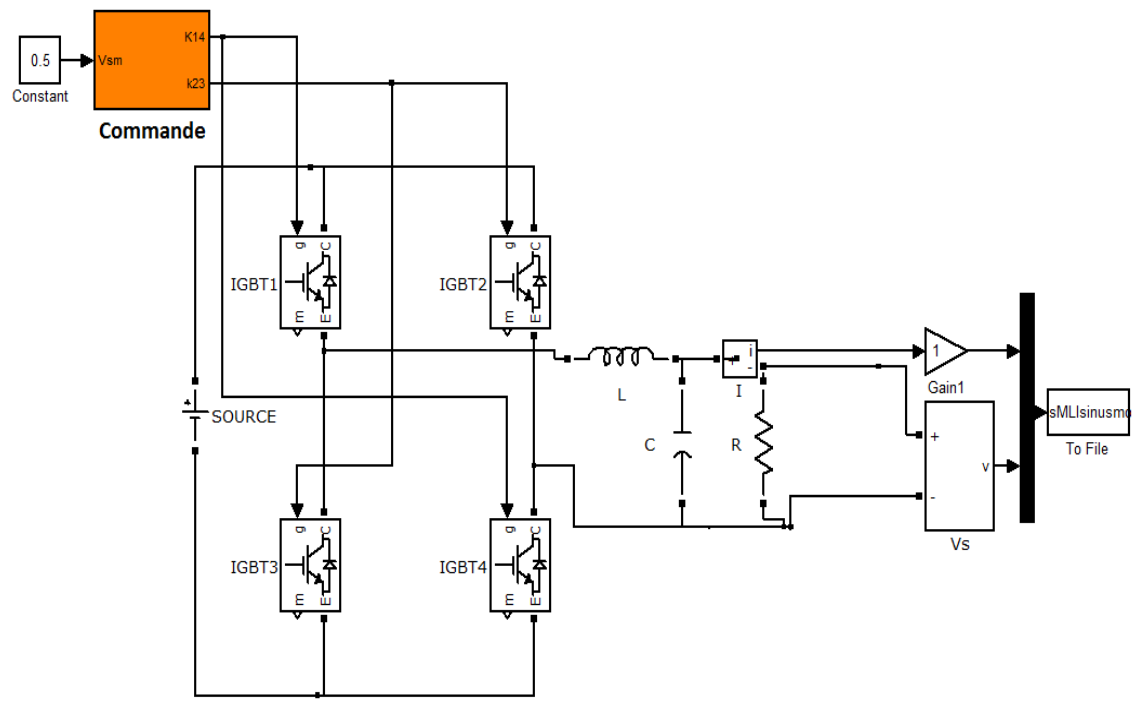

Fig. 7: Model simulation

$$
\frac{f_{p}}{f_{0}}=6 \mathrm{p}+3
$$

Having regard to the study of these control techniques, it is necessary to take certain parameters or comparison tools. The latter being sometimes the harmonic distortion factor, time to response or restoring the system as well as losses by switching the switches.

Distorsion factor: Traditionally, the rate of distortion harmonic voltage (THDv) or the inverter output current (THDi) is used to assess the quality of voltage or current of a PWM strategy. More the THD, more this strategy PWM contains harmonics of high amplitude.

However, the analytical calculation of the THDv requires a complex procedure.

It is therefore rather suited in the post-processing of the results of the numerical simulations (Bowes, 1975):

The rate of voltage harmonic distortion is expressed as a percentage of harmonics from the fundamental of a signal: where $\mathrm{Vn}$ is the amplitude of the harmonic of order $n$ and $V_{1}$ is the amplitude of the fundamental. Later, we use this criterion for digital simulation results:

$$
\operatorname{THD}(\%)=\frac{\sqrt{\sum_{n=2}^{\infty} V_{n}^{2}}}{V_{1}^{2}} \cdot 100
$$


Table 1: Parameters of simulation

\begin{tabular}{ll}
\hline $\mathrm{Vdc}(\mathrm{V})$ & 300 \\
\hline $\mathrm{F}(\mathrm{kHz})$ & 20 \\
$\mathrm{~L}(\mathrm{H})$ & $41,6.10^{-3}$ \\
$\mathrm{C}(\mathrm{F})$ & $200.10^{-6}$ \\
$\mathrm{R}(\Omega)$ & 25 \\
\hline
\end{tabular}

The inverter on Matlab/Simulink simulation: In the majority of cases of design and analysis of the performance of power electronic systems, it is often made call initially to simulators dedicated specifically to the analysis of the structures. Thus, at this stage, after a sizing of the filter, we present simulation results, carried out in the MATLAB/Simulink environment.

Table 1 parameters was used for the simulation of the model of Fig. 7 under Matlab-Simulink.

\section{RESULTS AND DISCUSSION}

The obtained simulation results enable us to obtain curves of voltage and current at the outlet of the inverter below (Fig. 8).

There is thus a sinusoidal waveform at the output of the inverter. So the response time is noted at the moment where the effective voltage remains constant from which a value of approximately $4,09.10^{-2}$ sfor the PWM Simple command.

The steady settled much more quickly than that noted for the PWM Simple command. The response time of approximately $\mathrm{t}=4 \cdot 10^{-2} \mathrm{~s}$ is viewable on Fig. 9. Note also that the study of harmonic content was assessed at the level of permanent regime.

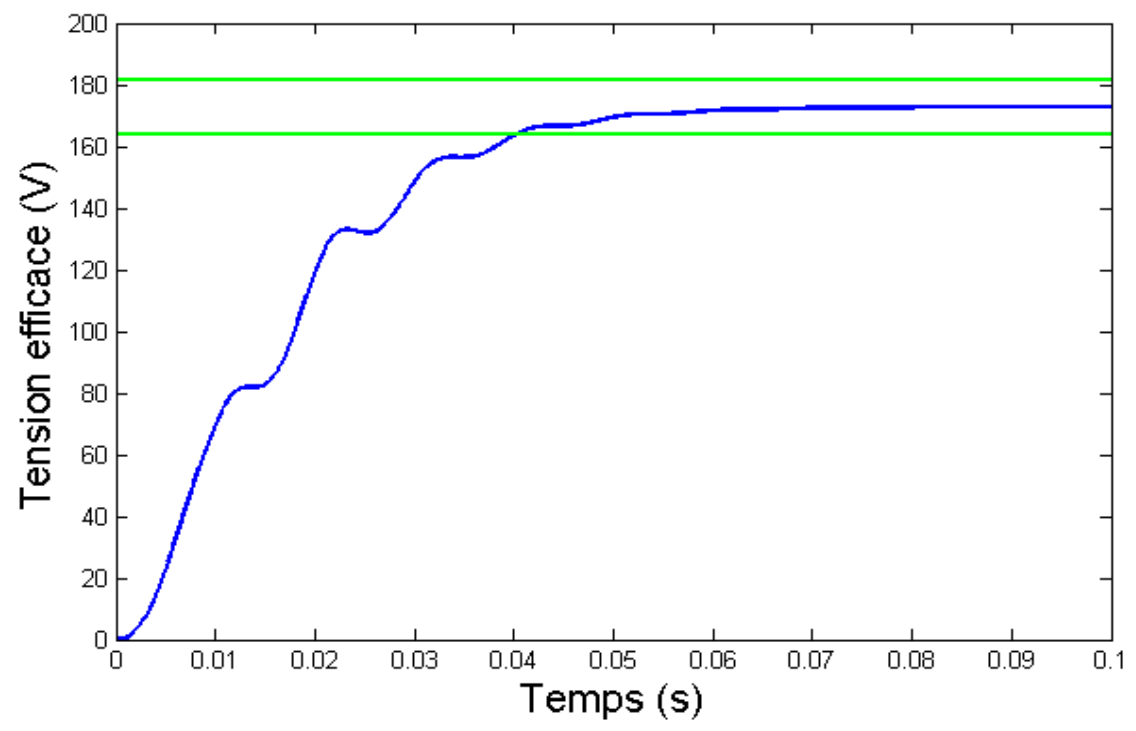

Fig. 8: Voltage and current for the simple PWM control

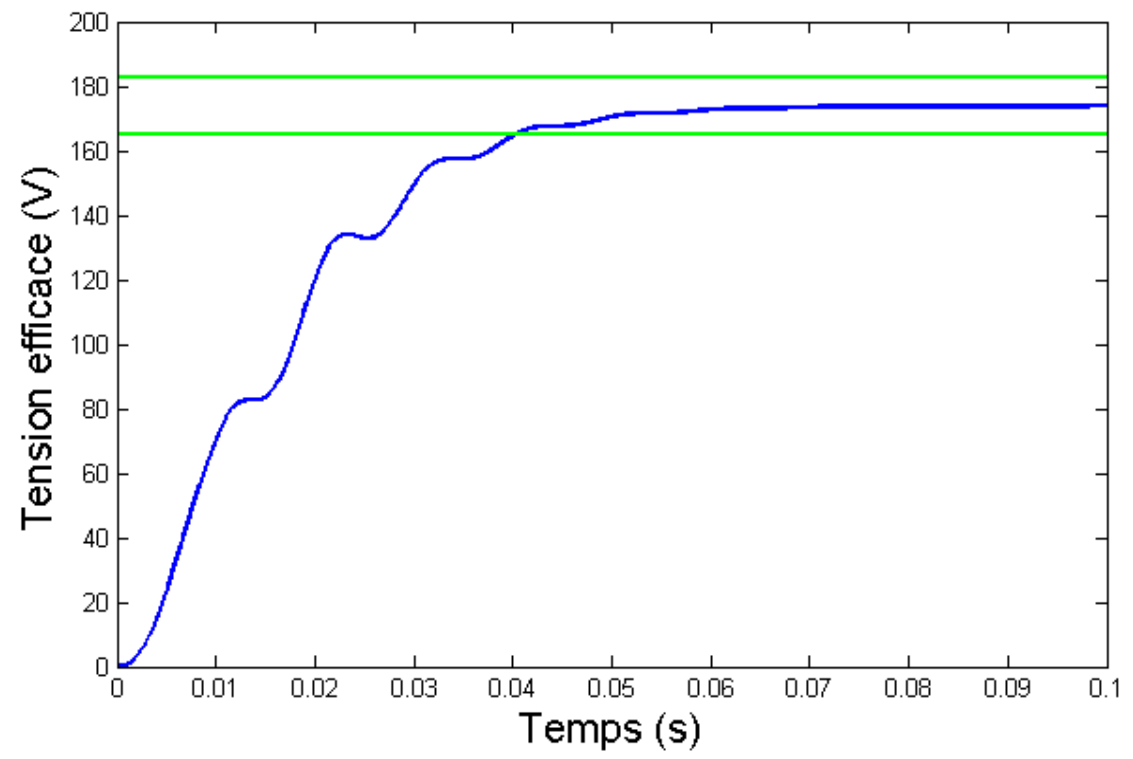

Fig. 9: Voltage and current output for multiple PWM control 


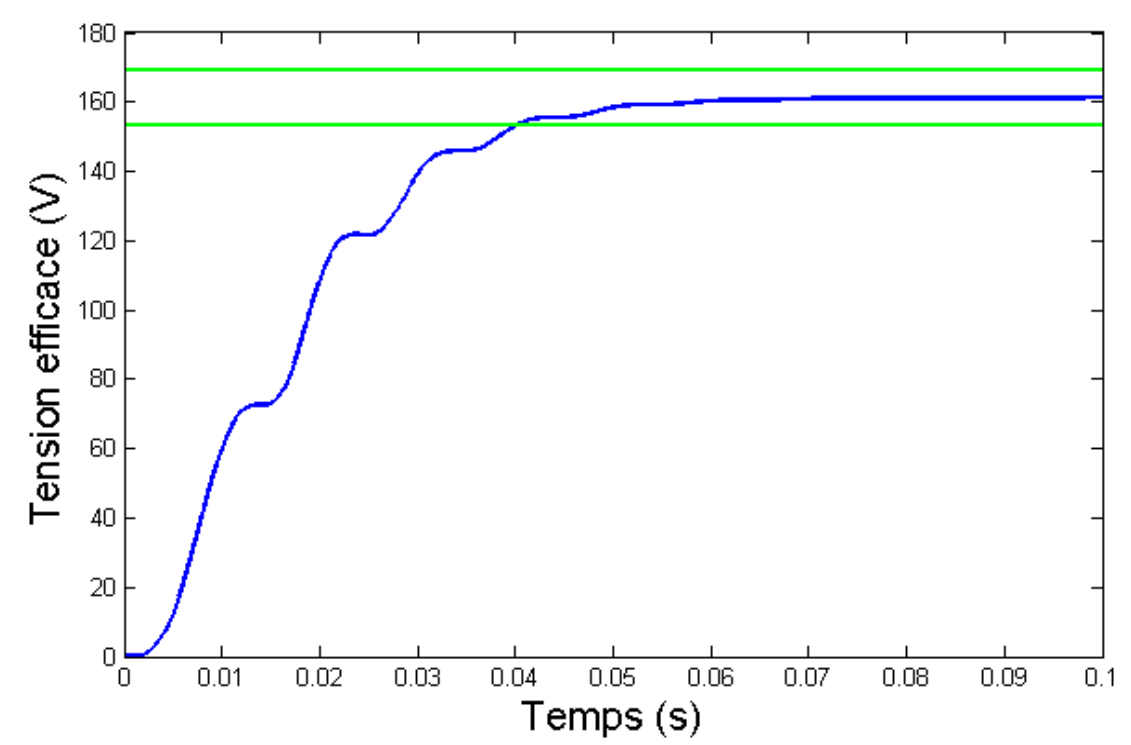

Fig. 10: Voltage and current output for sinusoidal PWM control

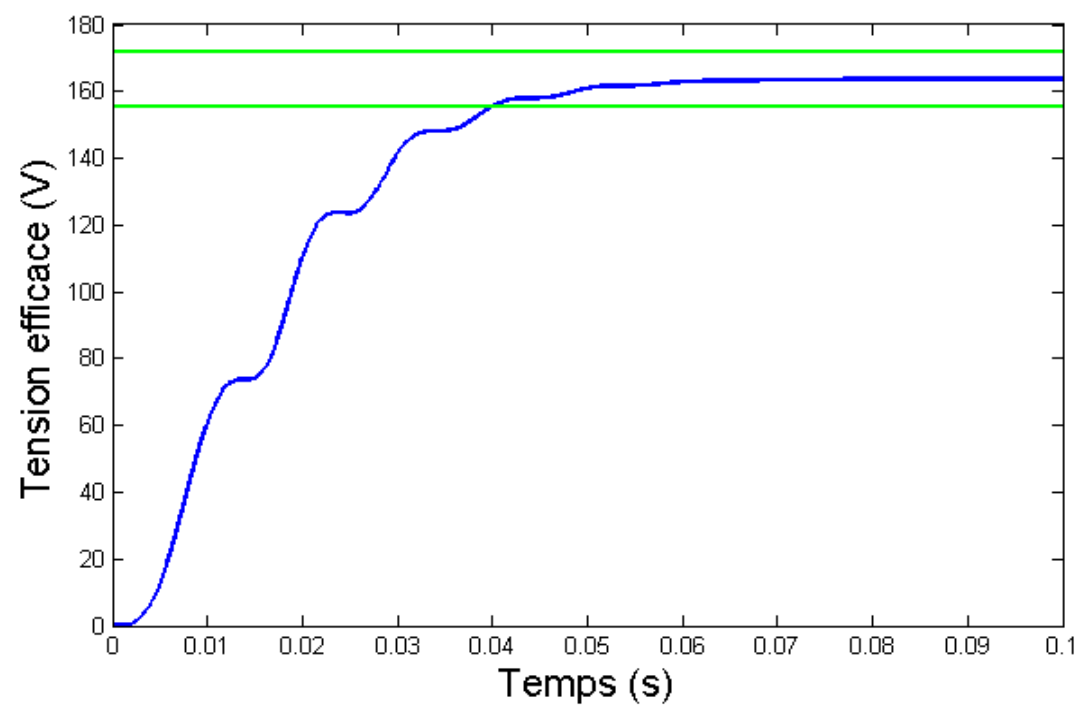

Fig. 11: Voltage and current to the sinusoidal PWM control modified

Study of the current and voltage waveform at the output of the inverter reveals a sinusoidal look as noted for other commands. But it is to make it clear that we have a gap no one between the current and the voltage as it is in resistive load, which is noted for all curves of different techniques. The response time of approximately $\mathrm{t}=3,93 \cdot 10^{-2} \mathrm{~s}$ is viewable in Fig. 10 .

However Fig. 11 allows us to say that we have a permanent regime establishes much faster than noted with the sinusoidal PWM command where a smaller response time of approximately $3,88.10^{-2} \mathrm{~s}$ (Fig. 12).

The current harmonics content shows that it is low side high frequency, it is observed that the amplitude of the fundamental current increases dramatically (Fig. 13). Greater the number of pulse (p), lower-order harmonic amplitude is small, but some higher-order harmonics is most important. However, they can be filtered.

Figure 14 shows that the filtering is excellent on the one hand, there is a sine wave on the other hand, harmonic distortion levels is improved because and the harmonic analysis of the current shows that the THD is reduced to a value of $8.39 \%$.

Thus it is noted that odd row stripes interfere with the (fundamental) main line, which was able to facilitate filtering. Hence the advantage of the sinusoidal PWM compared to the linear PWM. The modified sinusoidal PWM command to obtain the best results on the criteria of gain, the time of response and quality of the spectrum of the output current. Thus its fundamental component is increased and the harmonic 


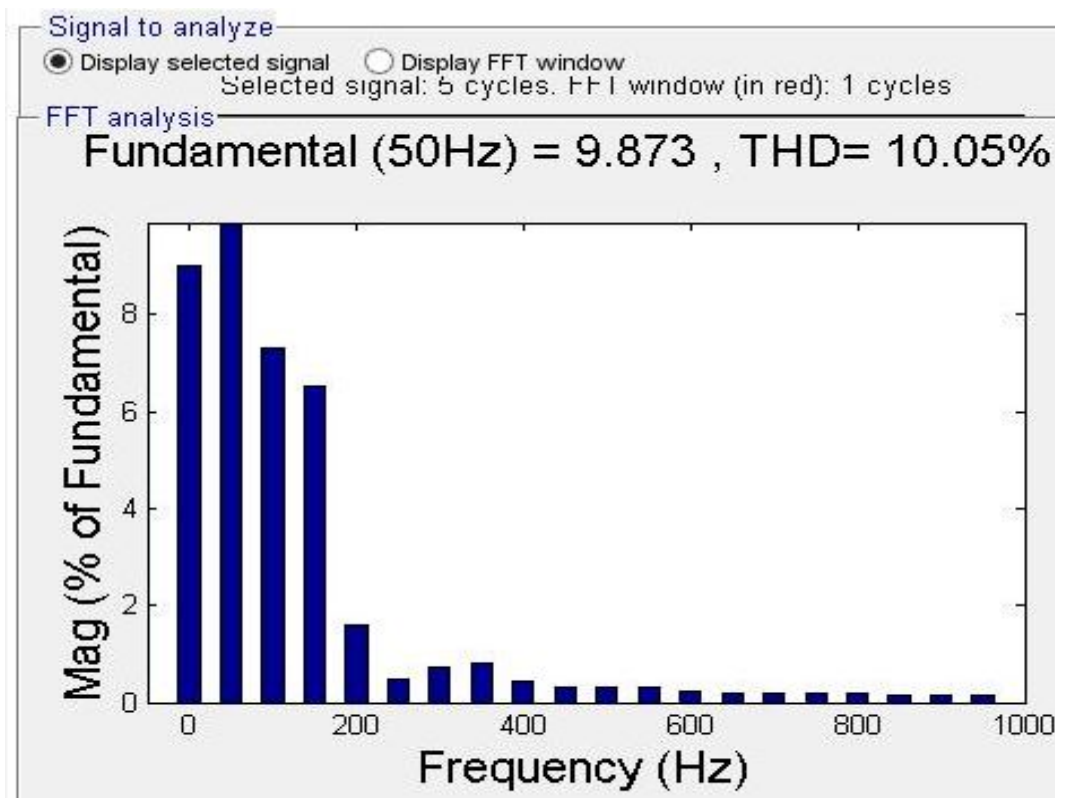

Fig. 12: Voltage and current for the simple PWM control

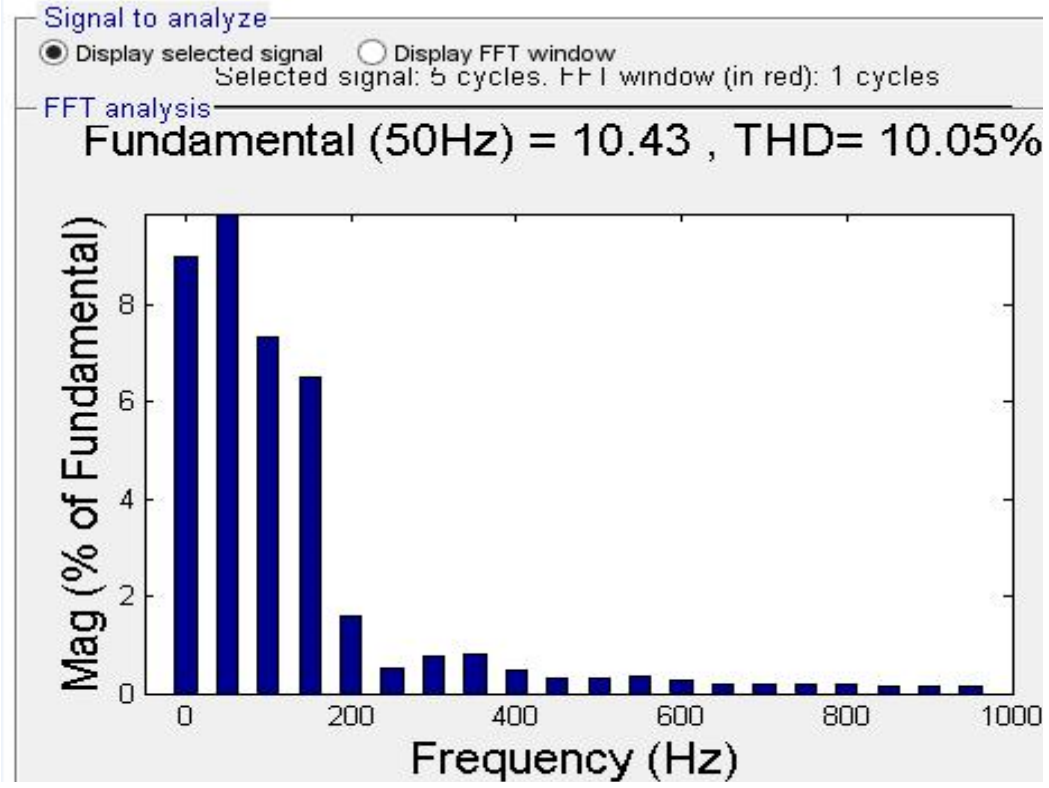

Fig. 13: Voltage and current for the multiple PWM control

spectrum is improved, which simplifies filtering advantage (Fig. 15).

Discussion: Comparative assessments of different commands are given. The maximum output voltage can be obtained for a given of the modulation index. Table 2 shows the harmonic profile for different commands. This shows that the modified sinusoidal modulation provides better harmonic profile as well as response time smaller The content in the current in the load harmonics depends strongly on the command applied to the inverter, it is noted that the harmonic distortion rate is slightly higher than in the case of the linear MLI (8.39\% for PWM sinusoidal and $10.05 \%$ for the MLI linear). The variation of voltage and current curves shows the relationship between the voltage stress (Vs) and the index of modulation $(, m-f)$ for different methods of control. It is clear that the voltage of the power switching devices stress is reduced when the modulation index is increased. Figure 12 to 15 show the effect of variation of the number of cycles of the wave of output as well as recovery time. In this case, we note that harmonic distortion is sensitive to these changes. For variations in the amplitude of the modulating and 


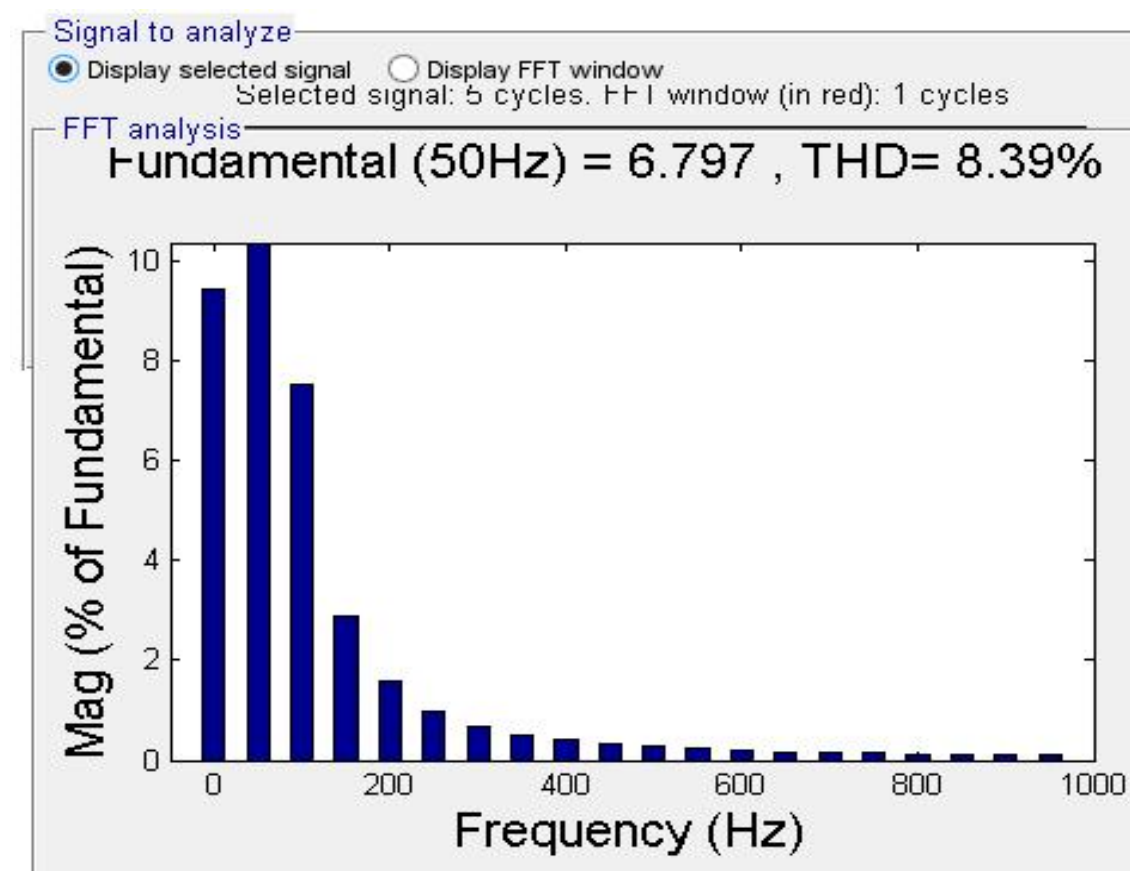

Fig. 14: Voltage and current for sinusoidal PWM control

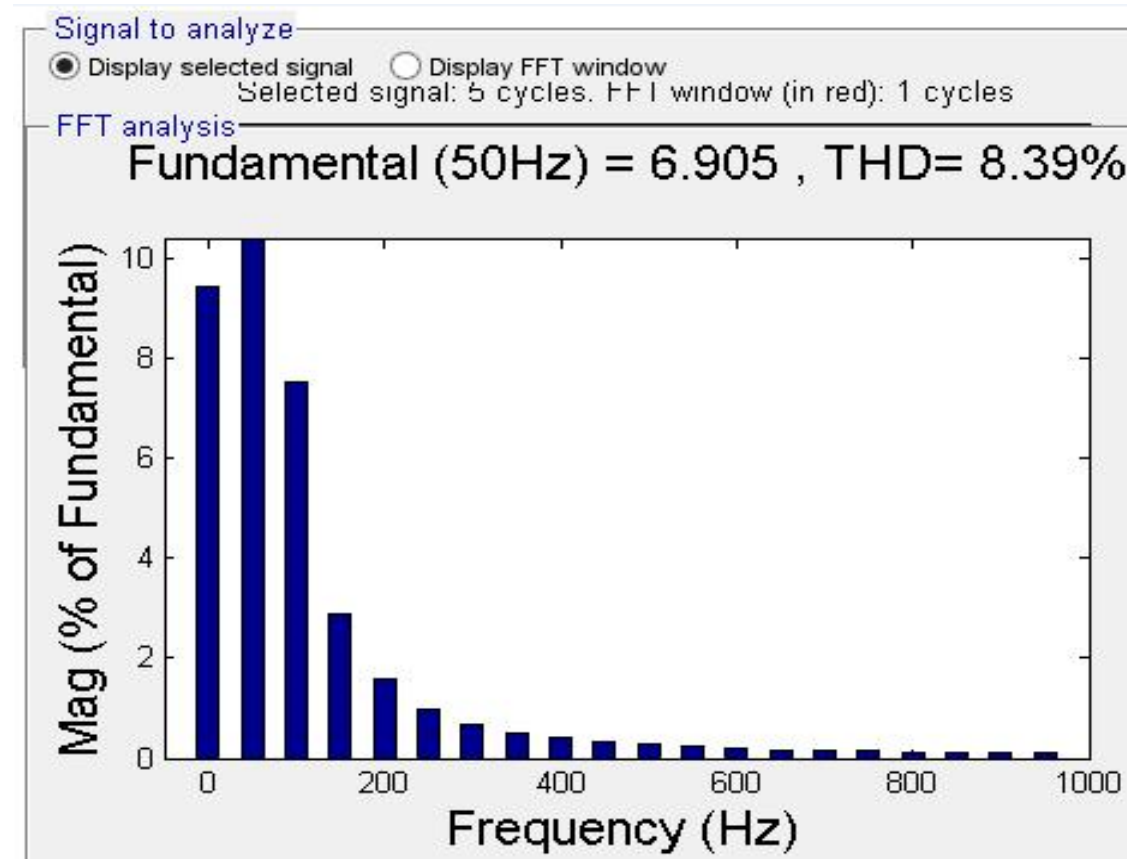

Fig. 15: Voltage and current for the modified sinusoidal PWM command

Table 2: Table of comparison of different commands

\begin{tabular}{llllll}
\hline Controls parameters & Simple PWM & Mutiple PWM & PWM sinusoïdal & PWM sinusoïdal & $\begin{array}{l}\text { PWM sinusoïdal } \\
\text { modified }\end{array}$ \\
\hline Current THD $(\%)$ & 10,05 & 10,05 & 8,39 & 8,39 & 8,39 \\
Response time $\left(10^{-2} \mathrm{~s}\right)$ & 4,09 & 4 & 3,93 & 3,93 & 3,88 \\
\hline
\end{tabular}

the reference frequency, one sees that converter perfectly follows these variations. The modified sinusoidal modulation technique allows operation best, when it is compared to all other methods of control. Note that we took during the simulation, an average response time of about $5 \cdot 10^{-2} \mathrm{~s}$ for all the various 
commands, hence the need to take the corresponding time of each command to see a wide variation of the THD.

\section{CONCLUSION}

In this document, four methods of modulation for a single-phase Inverter control have been examined and compared. The appropriate command mode can be selected in function of the requirement of different loads and applications. Simulations have been developed in the environment Matlab/Simulink for a single-phase inverter with one charge $\mathrm{R}$.

The result of spectral comparison shows that the modified sinusoidal pulse width modulation provides best performance with the lowest harmonic. On the basis of the comparative assessment, the relevance of the methods of control for different applications is also described.

Future work may be extended for the practical realization of the sinusoidal PWM command changed and comparison and implementation of control techniques of Multi Functions Grid Connected Inverter (MFGCI).

\section{REFERENCES}

Boumaaraf, A., M.D. Draou and S.A. Chikhi, 2002. Un nouveau concept de la commande PWM destiné au système photovoltaïque. Rev. Energ. Ren., 5: 139147.
Bowes, S.R., 1975. New sinusoidal pulsewidthmodulated invertor. P. I. Electr. Eng., 122(11): 1279-1285.

Chikhi, S. and F. Chikhi, 2002. Amélioration de la réponse spectrale du signal de sortie d'un pont monophasé par l'application des signaux de commande générés par la méthode triangulosinusoïdale, Décalés de $\pi$. Rev. Energ. Ren., 5: 3338.

Houldsworth, J.A. and D.A. Grant, 1984. The use of harmonic distortion to increase the output voltage of a three-phase PWM inverter. IEEE T. Ind. Appl., IA-20(5): 1224-1228.

Ryan, M.J., W.E. Brumsickle and R.D. Lorenz, 1997. Control topology options for single-phase UPS inverters. IEEE T. Ind. Appl., 33(2): 493-501.

Shete, P.S., R.G. Kanojiya and N.S. Maurya, 2012. Performance of sinusoidal pulse width modulation based three phase inverter. Proceeding of the International Conferece on Emerging Frontiers in Teccnology for Rural Area (EFITRA).

Thangaprakash, S. and A. Krishnan, 2010. Comparative evaluation of modified pulse width modulation schemes of Z-source inverter for various applications and demands. Int. J. Eng. Sci. Technol., 2(1): 103-115. 